


\section{WAHRNEHMUNG UND/ODER KOMMUNIKATIONP Neue Literatur zu Sport, Medien, Mediensport}

Besprochen von MARKUS STAUFF

Raymond Boyle, Richard Haynes, Power Play: Sport, the Media, and Popular Culture (2. erw. Auflage), Edinburgh (Edinburgh Univ. Press) 2009.

Eileen Kennedy, Laura Hills, Sport, Media and Society, Oxford, New York (Berg Publishers) 2009.

Tobias Werron, Der Weltsport und sein Publikum: Zur Autonomie und Entstehung des modernen Sports, Weilerswist (Velbrück) 2009.

Zeitschrift für Kulturphilosophie, (Schwerpunkt: Brot und Spiele) 4/r, Hamburg (Felix Meiner) 2010.

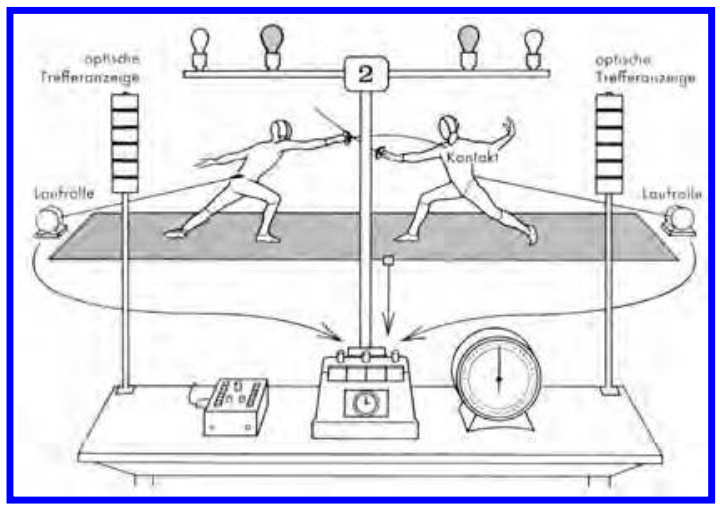

die überraschend persistente Relevanz des Sports dabei, gerät sowohl die konstitutive Bedeutung des Sports für Medien (oder zumindest für mediale Formen, Gattungen, Funktionen und Technologien) als auch, umgekehrt, die mediale Konstitution des modernen Sports differenzierter in den Blick - darauf weisen zumindest einige, wenn auch nicht systematisch aufeinander bezogene Aufsätze hin:

Erst kürzlich hat Victoria Johnson in einem instruktiven Beitrag gezeigt, warum und in welcher Weise der Sport über alle Transformationen des Fernsehens hinweg so bedeutsam ist ${ }^{2}$ : Die doppelte zeitliche Struktur des Sports (der sowohl verbindliche Live-Ereignisse als auch beliebig reproduzierbare Highlights hervorbringt) sowie seine doppelte Adressierung (Expertenwissen und Common Sense), unterstützen die kulturelle und ökonomische Logik der gegenwärtigen medialen Ausdifferenzierung. In einem Themenheft des Sociology of Sport Journal zu digitalen Medien (26/1 2009) setzen sich wiederum mehrere Aufsätze mit der Remediatisierung des Sports in 
Computerspielen auseinander und zeigen beispielsweise, wie der Sport zu einer spezifischen Gestaltung der Interfaces $^{3}$ oder zu einer spezifischen post-humanen Subjektivierung ${ }^{4}$ beiträgt. Entscheidende Impulse für eine solche Diskussion liefern auch die schon etwas älteren historischen Fallstudien von Richard Haynes zum Stellenwert des Sports in der frühen Geschichte der BBC (Radio und Fernsehen). ${ }^{5}$ Er arbeitet heraus, wie Sport genutzt wurde, um das Ideal vom Rundfunk als die Nation verbindende Technologie zu plausibilisieren - und wie dies zugleich dazu beitrug, bestimmten Ereignissen einen kollektiv verbindlichen Status zu verleihen:

[F]ootball, and more generally the whole field of television outside broadcasts from sporting events, were crucial to the manner in which the $\mathrm{BBC}$ sought to define what television actually was, and that within the initial throes of developing a television service there are some residual discourses which continue to pervade contemporary debates regarding televised football. ${ }^{6}$

Gemeinsam ist solchen sehr unterschiedlichen Aufsätzen, dass sie Sport und Medien zwar nicht pauschal gleichsetzen, aber die Medien auch nicht auf die Funktion einer bloß nachträglichen Repräsentation des Sports und den Sport nicht auf einen besonders erfolgversprechenden Gegenstand der Medien reduzieren; sie stellen die Frage nach den spezifischen kulturellen, ökonomischen, ästhetischen und technischen Dynamiken im Schnittfeld von Sport und Medien.

Vor diesem Hintergrund möchte ich im Folgenden einige aktuelle Publikationen zur Diskussion stellen: Eine soziologische Studie, die eine originelle und weitreichende Neuperspektivierung sowohl der historischen Entwicklung als auch der systematischen Erklärung des Zusammenhangs von Medien und Sport vorschlägt; zwei einführende Übersichtswerke zum Komplex Mediensport aus dem Umfeld der Cultural Studies sowie einen Themenschwerpunkt der Zeitschrift für Kulturphilosophie mit dem Titel Brot und Spiele.

Der Bielefelder Soziologe Tobias Werron geht in seiner Studie Der Weltsport und sein Publikum. Zur Autonomie und Entstehung des modernen Sports der schlichten Frage nach, wie der Sport - gemeint ist hier ganz explizit moderner Wettkampfsport und nicht die umfassendere Bewegungskultur - zu einem derart global erfolgreichen Phänomen werden konnte. Die Antwort ist zunächst nicht weniger schlicht, birgt aber nicht nur für die Beschäftigung mit dem Sport, sondern für die Medienwissenschaft allgemein ein erstaunliches Potenzial: Der moderne Sport hat sich historisch herausgebildet und reproduziert sich auch gegenwärtig noch als ein Bereich mit einer spezifischen Eigenlogik, weil er die Wahrnehmung des einzelnen (ggf. ästhetisch attraktiven) Wettkampfs in einen kommunikativ und somit medial etablierten, tendenziell universellen Vergleich von Leistungen einbindet. Die Vereinheitlichung von Regeln und die regelmäßige Veranstaltung von Wettkämpfen einzelner Sportarten, die in der (sport-) historischen Literatur häufig als entscheidende Faktoren angeführt werden, erhalten nur Plausibilität und Stabilität, weil sie sich mit einem dritten Faktor zu einer sich wechselseitig stützenden Konstellation zusammenschließen: der Etablierung eines sportspezifischen Publikums (inklusive eines entsprechenden (Gedächtnisses`), das sich nicht nur vom einzelnen Wettkampf, sondern von einem zeitlich, räumlich und auch sozial möglichst ausgeweiteten Leistungsvergleich faszinieren lässt.

In einem ersten, systematischen Teil werden die operativen Bedingungen (und somit auch die medialen Verfahren) beschrieben, «die dem Wettkampfverhalten kommunikative Qualität verleihen und zugleich Deutungszusammenhänge zwischen einzelnen Wettkämpfen begründen» (S. 59). Im Detail schildert Werron wie quantitative (Ergebnisse, Statistiken etc.), visuelle (Tabellen, Grafiken etc.) und narrative (Charaktertypologien, Spielverläufe etc.) Darstellungsformen die Komplexität von Wettkämpfen zunächst so reduzieren, dass Teilereignisse jenseits des bloßen Endergebnisses als Leistungen identifizierbar, auf unterschiedliche Faktoren zurechenbar und letztlich auch vergleichbar werden. Diese Umwandlung der Wahrnehmung von einzelnen Wettkämpfen in kommunizierbare Einheiten trägt damit zugleich zum Komplexitätsaufbau bei, weil bei der künftigen Wahrnehmung eines Wettkampfs dessen Teilereignisse in einen sehr viel umfassenderen Sinnhorizont eingebettet werden.
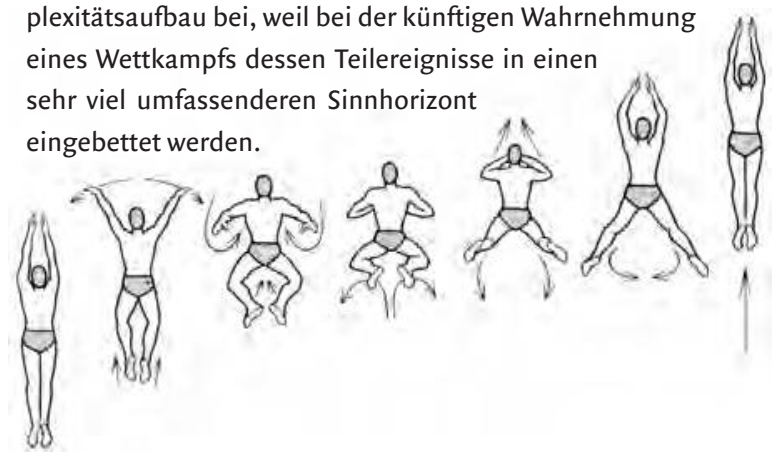


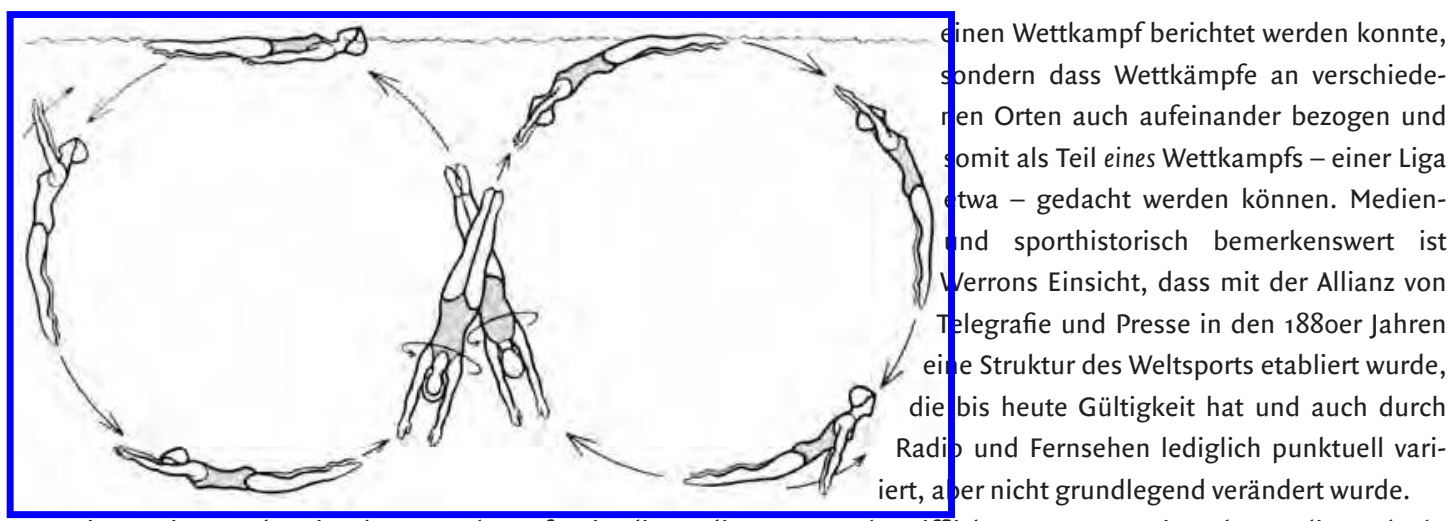

Die Kontingenz des einzelnen Wettkampfs reizt die mediale Zuschreibung und Evaluation von Leistung fortlaufend neu an; die dadurch ermöglichte differenzierte Klassifizierung von Leistungen erlaubt eine weitere Steigerung der Kontingenz - und sei es nur durch die Organisation von Setzlisten oder die Spekulation über «wahrscheinliche> Wettkampfverläufe. Angesichts der Vielzahl der unterschiedlichen Sportarten und der Heterogenität der eingesetzten medialen Verfahren eröffnet sich hier ein noch weitgehend unerschlossenes Feld für die weitere Forschung, könnten doch etwa die in der Wissenschaftsforschung diskutierten Fragen nach den wissenskonstitutiven Effekten von Darstellungsformen in einem ganz anders strukturierten Praxisfeld aufgegriffen werden.

Im zweiten, historischen Teil rekonstruiert Werron die Entstehungsprozesse von Fußball in England und Baseball in den USA im 19. Jahrhundert - immer wieder ergänzt durch Vergleiche mit weiteren Sportarten wie Schach oder Boxen. Überzeugend weist er nach, dass es vor allem der Verbund von Massenpresse und Telegrafie war, der einen Horizont für den Vergleich von Leistungen über den einzelnen Wettkampf hinaus eröffnete: «Durch die Aufzeichnung in der telegraphisch gestützten Presse wird der zeitnahe Vergleich vieler räumlich distanzierter Wettkämpfe im Horizont des Publikums erstmals denk- und realisierbar.» (S. 231) Dies ist keine mediendeterministische, monokausale Erklärung: Immer wieder wird der zirkuläre Zusammenhang zwischen der zunehmenden Standardisierung der Regelwerke, der zunehmend kontinuierlichen Veranstaltung von Wettkämpfen und der Etablierung eines Publikums, das die an verschiedenen Orten und zu verschiedenen Zeiten erbrachten Leistungen vergleicht, betont. Die Vernetzungsweise der Telegrafie macht es dabei nicht nur möglich, dass schnell (manchmal - v.a. im Fall des grafisch notierbaren Baseballs - sogar live) über
Das begriffliche Instrumentarium der Studie verdankt sich - wie ja die Ausgangsfrage nach der historischen Ausdifferenzierung einer Eigenlogik des Sports schon nahelegt - der Systemtheorie. Diese erweist sich hier als äußerst dienlich, um Problemstellungen zu schärfen und gewisse vermeintliche Evidenzen zu unterlaufen. Prägnant ist etwa, wie Werron einerseits darauf insistiert, dass der globale ‘Erfolg) des Sports weder ökonomisch noch politisch, sondern nur durch die Verfahrensweisen des Sports selbst erklärt werden kann, wie er aber andererseits sehr präzise beschreibt, dass ein System, das in diesem Ausmaß durch öffentliche Beobachtung und Evaluierung konstituiert wird, hohes Attraktions- und Verwertungspotenzial für Politik und Wirtschaft, für Lifestyle und vielfältigste Ideologien hat.

In ihrer immer sehr prägnanten Argumentation verbindet die Studie zahlreiche interessante Details der Sportgeschichte zu vielen überraschenden Einsichten. Beeindruckend ist die umfassende Berücksichtigung von soziologischer, medienwissenschaftlicher, historischer und sportwissenschaftlicher Literatur; besonders erfreulich ist der analytisch überzeugende Einbezug der Formen und Semantiken des Mediensports - seien es die Sportzeitschriften des 19. Jahrhunderts, die grafischen und statistischen Aufbereitungen auf Webseiten oder die Autobiografien von Sportlern und Funktionären. Aus medienwissenschaftlicher Sicht liegt die Stärke des Bandes neben der Auseinandersetzung mit medialen Formen der Leistungsevaluation und der historischen Neuperspektivierung der Telegrafie nicht zuletzt darin, dass eine präzise Modellierung des Verhältnisses von Sport und Medien formuliert wird, die der auch in der Wissenschaft verbreiteten pauschalen Behauptung (bzw. Kritik an) einer zunehmenden Mediatisierung (und ‘spektakularisierung)) des Sports den Boden entzieht. 
Zugleich versteht Werron seine Studie völlig zu Recht auch als einen Beitrag zur Globalisierungsforschung: Historisch zeigt er, wie Klassenzugehörigkeit, pädagogische oder nationalistische Ideologien in die Begründung des Sports involviert waren, wie sie dabei aber Verfahren etablierten, die auf Universalität zielten; Verfahren die im Übrigen den lokalen Wettkampf und damit auch die lokale Identifikation als Ressource dringend brauchen (und reproduzieren), sie aber zugleich durch den globalen Vergleichszusammenhang dekontextualisieren: «Der moderne Sport entsteht, indem er sich die Potenziale der Begrenzung der Wettkampfform durch Transzendierung dieser Grenzen zu nutze macht.» (S. 57)

Die Relevanz dieser Argumente wird im Blick auf andere aktuelle Publikationen noch verstärkt. Der Themenschwerpunkt Brot und Spiele der Zeitschrift für Kulturphilosophie (Ausgabe 1, 2010) beispielsweise interessiert sich ebenfalls für die "Sonderwelt» (S.6) des Sports, die, wie die kurze Einleitung unterstreicht, kaum geeignet ist, «eine Moral zu entfalten oder überhaupt so etwas wie eine Botschaft zu enthalten» (ebd.). Obwohl sie sich zumindest implizit auf modernen Leistungssport beziehen, fokussieren durchgängig alle Beiträge allein die Seite des unmittelbaren Wettkampfs. Damit greifen sie vor allem Aspekte von Wahrnehmung sowie von (präsenter) körperlicher Bewegung auf-Kommunikation und Medialisierung von Sport sowie die dadurch mögliche Relationierung voneinander räumlich oder zeitlich getrennter Leistungen und Wettkämpfe spielen keine Rolle.

In einigen der Beiträge lässt sich dies als Fokussierung eines für bestimmte philosophische Fragen möglicherweise besonders prägnanten Aspekts des Sports verstehen: Volker Schürmann etwa sieht im Sport eine Herausforderung für die Hermeneutik, insofern die Wettkämpfe (entgegen der Behauptung einer kompletten Bedeutungslosigkeit) durchaus etwas darstellten - aber eben nichts außerhalb des Sports, sondern allein den Sinn «jenes gegenwärtig vollzogenen Treibens» (S. 58). Es sei somit Aufgabe einer Praxis-Philosophie herauszuarbeiten, wie der Vollzug der sportlichen Bewegungen weniger das Resultat als vielmehr die Voraussetzung von Verstehen ist. Elk Franke diskutiert die ethischen Implikationen des Sports und stellt dabei heraus, dass dieser keinerlei situationsübergreifende Urteilsbildung (etwa im Sinne der seit dem 19. Jahrhundert etablierten Idee, er könne zu Fairness erziehen) schafft. Gleichzeitig arbeitet Franke unter Rückgriff auf ein vertragstheoretisches Vokabular aber heraus, dass eine gegenstandsspezifische Sportethik sehr wohl existiert, die sich vor allem durch die (zumindest immer zu unterstellende) Akzeptanz von in Alltagssituationen sinnlosen Handlungsbedingungen bildet. Volker Caysa schließlich diskutiert die Frage, wie es auf der Basis einer «empraktischen Könnerschaft», d.h. eines routinisierten und automatisierten «wissenden Nichtwissens», zur «außerordentlichen Lösung einer Aufgabe» (S. 69) im Wettkampf und somit zu Neuerungen kommen kann.

In allen drei Beiträgen wirkt sich die Nichtberücksichtigung der medialen Konstitution von Sport allerdings beschränkend auf die jeweiligen Grundkonzepte aus: Caysa etwa führt innovative körperliche Lösungen auf innere Bildproduktion zurück, die bei ihm zwar explizit «mit allen fünf Sinnen» in Verbindung steht, aber eben nicht mit den vielfältigen Visualisierungstechnologien, die schon seit vielen Jahrzehnten nicht nur die Rezeption, sondern auch die Produktion von Sport (also Training, Strategieentwicklung, Selektion von AthletInnen) grundlegend mit strukturiert. ${ }^{7}$ Die Ausblendung dieser Faktoren führt zu einer sehr traditionellen Konzeption vom individuellen und autonomen Körper, die sich ähnlich im Beitrag Frankes zeigt: Er beschreibt zunächst völlig plausibel, dass ein spezifischer Reiz des Sports darin besteht, die Leistungen komplexitätsreduzierend Individuen zuschreiben zu können, leitet daraus aber als eine der impliziten Vertragsbestandteile ein "Natürlichkeitsversprechen" der Wettkampfteilnehmer ab. Statt also die medialen Verfahren zu analysieren, die auf der einen Seite die körperliche Leistung mit produzieren, auf der anderen Seite aber (durch Statistiken, Zeitlupen, Narrative etc.) eine immer offene, immer dynamische Zurechnung der erbrachten Leistung ermöglichen, wird ein Effekt dieser Zurechnungsprozesse - die «natürlichen Veranlagungen des Handelns" (S.24) - zu einem Wesensmerkmal des Sports. Parallel zu dieser «Naturalisierung〉 des Körpers als fundamentaler Bezugsgröße wird in Schürmanns Beitrag der einzelne Wettkampf durch die Betonung seines kontingenten Verlaufs idealisiert: Das ihm zufolge zen- 
trale prozesshafte Verstehen im Vollzug der Bewegung werde zugespitzt durch die Unvorhersehbarkeit von Sieg und Niederlage, sodass ein gelungener Wettkampf «die gelungene Inszenierung der Wettkampfgegner als gleichwertige» (S. 64) impliziere. Auch hier wird ausgeblendet, dass die «Gleichwertigkeit der Gegner` nicht zuletzt durch die Organisation von hierarchisierten Ligen und Rankings immer wieder neu - und gewissermaßen ‘jenseits` des einzelnen Wettkampfs - produziert werden muss und dass darüber hinaus die UUnvorhersehbarkeit von Sieg und Niederlage - und generell die den Sport kennzeichnende Kontingenz - zugespitzt wird durch die wiederum auf Basis von Statistiken, Narrativen und anderen medialen Verfahren produzierten Erwartungsformulierungen und Vergleichsmöglichkeiten.

Ganz ähnlich der Beitrag von Hans Ulrich Gumbrecht: Er zeigt sehr schön, wie Sport mit den beiden Grenzbereichen von Kontingenz - Notwendigkeit und Unmöglichkeit - spielt; durchaus nachvollziehbar gliedert er die Sportarten in sechs unterschiedliche Typen von Kontingenzproduktion und versucht (etwas zu kursorisch) ihren historisch variablen Erfolg mit den in der Gesellschaft je dominanten Konzepten von Kontingenz zu begründen. Es bleibt aber auch hier ein Schwachpunkt, dass nicht diskutiert wird, wie der mediatisierte Leistungsvergleich für alle Sportarten ähnliche Verfahren der Kontingenzproduktion (Weltmeisterschafen, Ranglisten, zu brechende Rekorde etc.) bereitstellt.

In anderen Beiträgen, die explizit aufs Ganze des Sports zielen, zeigen sich die Defizite der Medienblindheit noch viel schärfer, insofern sie zu einem (methodologisch und theoretisch) höchst konservativen Körperkonzept führen und weitab von einer philosophischen Perspektivierung des tatsächlich existierenden Sports zu einer eigenartig normativen Ästhetik führen. Am deutlichsten ist dies, nicht ganz unerwartet, in den Beiträgen von Martin Seel und Gunter Gebauer, in denen Medien dann auch nur gemeinsam mit 'Kommerzialisierung) in warnenden Nebensätzen auftauchen: Seel behauptet, dass nur ein Rückgriff auf die Ästhetik das Dopingverbot begründen könne. Sein zentrales Argument hierbei ist, dass Doping die für die Ästhetik des Sports zentralen Aspekte der Eigenleistung und der Unvorhersagbarkeit unterlaufe. Bezugspunkt ist dabei Kants Spielbegriff und die daraus abgeleitete Behauptung, dass dem sportlichen Sinn «auf Seiten der Akteure ein Geschehenlassen entspricht» (S. 14) und dass Sport so das Ritual all derer sei, «die sich Zeit da- für nehmen, an der eigenen Vergänglichkeit Gefallen zu finden» (S.12). Hier stellt sich zum einen die Frage, auf welcher Basis (und mit Bezug auf welchen Sport) diese Aussagen getroffen werden (Seels schlichte Herleitung des «Geschehenlassens" aus Empfehlungen an Athleten und Athletinnen, ssich nicht verrückt machen zu lassen», ließe sich ja mit dem nicht weniger verbreiteten socus, focus, focus> kontern). Zum anderen sind dies kaum plausible Argumente gegen Doping - entgegen Seels These, dass Doping dem (vermeintlich antisportlichen) Wahn einer "krisensicheren Leistungsfähigkeit» entspringe, wird solche Krisensicherheit im gegenwärtigen Sport in allererster Linie durch mental coaching u. a. psychologische Hilfestellungen zu erreichen versucht. Seel will deutlicherweise den Sport als Bereich des individuellen, unversehrten und autonomen Körpers retten, wenn er unterstellt: «Wir wollen die Körper der Athleten nicht als Repräsentanten pharmazeutischer Produkte, sondern in ihrer individuellen und also unberechenbaren Bewegtheit wahrnehmen können.» (S. 1of.)

Ein etwas zeitgenössischerer Körper- (und agency-) Begriff müsste demgegenüber fragen, inwiefern schon ein Wecker, der es den Athleten und Athletinnen möglich macht, ihr Training um 6 Uhr früh aufzunehmen, die eindeutige Zuschreibung der «antrainierten Eigenleistung» (S.10) kompliziert. Wenn Seel schließlich insistiert, dass «sich das Erstaunliche am sportlichen Gelingen ohnehin jeder Statistik entzieht»(S.11), wird überdeutlich, dass sein von den Konzepten Körper und Spiel dominiertes Sportverständnis wenig mit dem modernen Sport zu tun hat. Zudem unterstellt es den unzähligen Fans, die sich von Statistiken, von Rekorden oder von Geschichten des «Nicht-einfach-Geschehenlassens〉 faszinieren lassen, eine nicht-sportgemäße Wahrnehmung. Gunter Gebauers Beitrag entfernt sich ähnlich weit von einer Reflexion existierender Wahrnehmungs- und Kommunikationsformen des Sports, wenn er mit Bezug auf die Antike und Foucaults Konzept der ‘Sorge um sich» konstatiert, dass der Sport den AthletInnen «die Freiheit zur Gestaltung eines athletischen Oeuvres" (S. 45) biete - dass dieses Potenzial in der Moderne aber zunehmend gefährdet sei: 
«Mit dem Glauben an die bloße Zahl und an die Anerkennung durch die Öffentlichkeit verlieren sie ihre Urteilsfähigkeit und Wertschätzung der Qualität ihrer Existenz. Sie verlieren die Freiheit zu entscheiden, was für sie wirklich von Bedeutung ist.» (S. 47$)^{8}$

Die Beiträge des Schwerpunkts, im Übrigen ausschließlich von männlichen Autoren, zeigen meines Erachtens noch durch ihre Defizite, wie unerlässlich die Berücksichtigung medialer Faktoren auch für eine Ästhetik des Sports sind - will diese sich nicht komplett von einer Reflexion des tatsächlich existierenden Sports entfernen. Dass alle Beiträge hier Sport vom Körper und vom Spiel bzw. zumindest vom einzelnen Wettkampf aus definieren, ist eine starke Selbstbeschränkung sportphilosophischer Reflexion. Zumal tatsächlich keiner der Beiträge internationale Literatur zu Geschichte und Ästhetik des Sports berücksichtigt, stellt sich die Frage, inwiefern sich hier (analog zur medienwissenschaftlichen Diskussion) ein ‘deutscher Sonderweg» bemerkbar macht.

Gewissermaßen spiegelbildlich zu den Beiträgen in der Zeitschrift für Kulturphilosophie funktionieren zwei englischsprachige Einführungen zum Mediensport, insofern deren Bezugspunkt von vornherein die medialen Repräsentationen von Sport sind; beide beschäftigen sich mit Fragen der Kommerzialisierung (Sponsoring, Branding), mit den Regelhaftigkeiten des Sportjournalismus, mit der Artikulation von race, class, gender, nation im Mediensport und mit Fankulturen.

Der Band Power Play: Sport, the Media, and Popular Culture von Raymond Boyle und Richard Haynes ist eine Neuauflage des 2000 erstmalig erschienenen Bandes, die jetzt um Kapitel zu Globalisierung und vor allem zu den «neuen` Medien ergänzt ist. Der Anspruch liegt nicht darin, eine bestimmte These oder Perspektive zu verdeutlichen, sondern darin, den Stellenwert des Sports in der gegenwärtigen Kultur in möglichst vielen Facetten zu beleuchten. Entsprechend gibt es - neben Kapiteln zu verschiedenen Aspekten der Repräsentation - auch Kapitel zur Geschichte der Medienberichterstattung über Sport, zu Sponsoring, zu Sportstars etc. Die Argumentation erfolgt entlang detailreicher Fallbeispiele - deren Zusammenhang aber manchmal undeutlich bleibt.

Der Band Sport, Media and Society, herausgegeben von Eileen Kennedy und Laura Hills, greift weitgehend ähnliche Themen auf, ist aber entlang einzelner Medien (Film, Fernsehen, Tagespresse, Magazine, Werbung, Internet) organisiert und hat eine noch stärker didaktisch einführende Struktur: In jedem Kapitel werden analytische Kategorien (Semiotik, Narrativität, Diskurs, Wissen etc.) vorgestellt und dann an je zwei oder drei Fallstudien, die jeweils auch die Aspekte race, class, gender, nation einbeziehen, zur beispielhaften Anwendung gebracht. Dadurch wird die Vielfalt der medialen Inszenierungen und somit auch das Zirkulationspotenzial deutlicher als im Band Power Play, der beispielsweise auf Fotografie gar nicht eingeht. Hervorzuheben ist etwa ein formale Analyse von zwei Fußballübertragungen (einmal englische Männer-, einmal englische Frauennationalmannschaft), die zudem mit Buscombes Analyse aus den 1970er Jahren (s.o. Fußnote 1) verglichen wird und so aufzeigt, dass es ein stabiles Formenrepertoire gibt, welches zugleich aber Variationen zulässt, die die Ernsthaftigkeit und Körperlichkeit des Männerspiels unterstreichen.

Beide Bände vermeiden starke Thesen zu dem spezifischen Zusammenhang von Medien und Sport; meist wird relativ vage ein symbiotisches Verhältnis unterstellt, das sich aus Faktoren wie excitement, drama, stars etc. ergibt. Am interessantesten ist hier sicher die Frage, inwiefern Sport und Medien je spezifische Zuschauergruppen definieren, von denen sie gegenseitig profitieren oder um die sie konkurrieren können. Beide Bände beginnen mit impressionistischen (wenn auch je anderen) Verweisen auf die Olympischen Spiele in Peking und begründen damit die kulturelle oder soziale Relevanz des Sports - wie zahlreiche ähnliche Bände ${ }^{9}$ - vor allem mit der quantitativ übermächtigen Präsenz des Sports in der gegenwärtigen Medienkultur.

Inwiefern der Sport ein spezifisches Strukturierungspotenzial für die Medien und der Mediensport ein besonderes Strukturierungspotenzial für kulturelle Formen und Semantiken haben, wird dabei kaum explizit diskutiert. Am ehesten werden solche Fragen noch in den Analysen von race, class, gender, nation aufgegriffen: Beide Bände

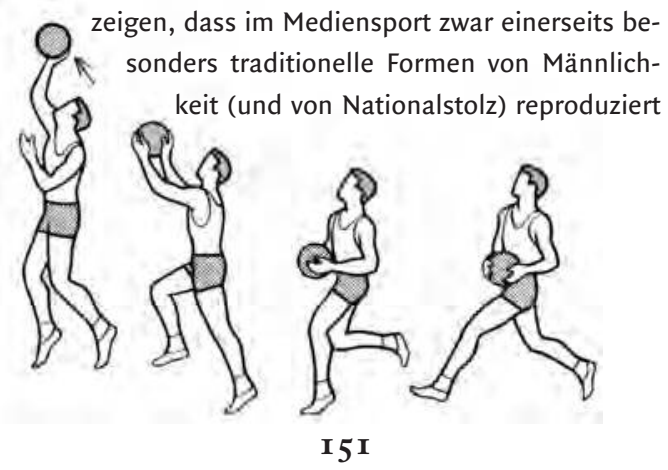




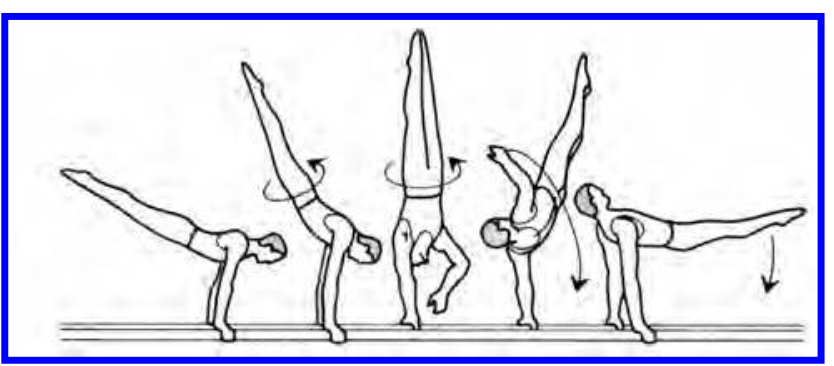

werden, dass aber andererseits zunehmend explizit ein weibliches Publikum adressiert werden soll - was wiederum bestimmte Pauschalisierungen der vermeintlichen ‘Vorlieben` von Frauen impliziert. Wie es aber genau zu solchen Strukturierungsleistung kommt, wird kaum rekonstruiert. Symptomatisch dafür ist auch, dass interne Differenzierungen des (Medien-)Sports nicht auf ihre Produktivität hin befragt werden; so wird beispielsweise die unterschiedliche Organisation von Raum und Zeit, von Teams und von Wettkämpfen, die die unterschiedlichen Sportarten voneinander unterscheidet, nicht diskutiert - vor allem in Power Play bleibt einmal mehr Fußball (wiederum aus Gründen der Quantität) der dominante Bezugspunkt.

Während die Bände somit in ihren Fallstudien zahlreiche Einsichten liefern, eröffnen sie doch zugleich wenig neue Perspektiven auf die Dynamiken des Mediensports. Spiegelbildlich zu den Beiträgen der Zeitschrift für Kulturphilosophie thematisieren sie Sport ganz aus einer etablierten Auffassung von Massenmedien her. Die Leistungen, die der Sport für die Medien erbringt, und die Relevanz des Mediensports für die gegenwärtige Kultur lassen sich weder mit Blick auf Spiel und Körper noch mit Blick auf die ökonomischen, technischen und formalen Mechanismen der Massenmedien umfassend erklären. Einmal mehr scheint es hier relevant, jenseits der vermeintlichen Selbstverständlichkeiten zunächst neue Fragen zu stellen.
1 Eine der zentralen Bezugspublikationen der weiteren Diskussion ist bis heute: Garry Whannel, Fields in Vision: Television Sport and Cultural Transformation, London, New York (Routledge) 1992; eine noch frühere, vorwiegend formale Analyse findet sich in: Edward Buscombe (Hg.), Football on Television, London (British Film Institute) 1975.

2 Victoria E. Johnson, Everything New is Old Again. Sport Television, Innovation and Tradition for a MultiPlatform Era, in: Amanda Lotz (Hg.), Beyond Prime Time: Television Programming in the Post-Network Era, London u. a. (Routledge) 2009, 114-137.

3 Steven Craig Conway, Starting at "Start»: An Exploration of the Nondiegetic in Soccer Video Games, in: Sociology of Sport Journal, 26/1, 2009, 67-88.

4 Darcy Cree Plymire, Remediating Football for the Posthuman Future: Embodiment and Subjectivity in Sport Video Games, in: Sociology of Sport

Journal, 26/1, 2009, 17-30.

5 Richard Haynes, «There's Many A Slip Twixt, the Eye and the Lipn: An Exploratory History of Football Broadcasts and Running Commentaries on BBC Radio, 1927-1939, in: International Review for the Sociology of Sport, 34/2, 1999, 143-156; ders., A Pageant of Sound and Vision: Football's Relationship with Television, 1936-196o, in: The International Journal of the History of Sport, 15/1, 1998, 211-226.

6 Haynes, A Pageant, 212.

7 Eine beeindruckende Übersicht zum Fußball aus journalistischer Perspektive findet sich bei: Christoph Biermann, Die Fußball-Matrix. Auf der Suche nach dem perfekten Spiel, Köln (Kiepenheuer \& Witsch) 2010.

8 Er folgt damit zugleich einer problematischen Lektüre des «Spätwerks' von Foucault, die Foucaults Freiheitsbegriff enthistorisiert, vgl. dazu: Swen Seebach, Robert Feustel, Freiheit im Vollzug. Foucaults Vorlesungen von 1978/79. Eine Replik auf Philipp Sarasin, in: Deutsche Zeitschrift für Philosophie, 56/1, 2008, 152-154 (diesen Hinweis verdanke ich Andrea Seier)

9 Etwa dem (theoretisch etwas profilierteren) Band: David Rowe, Sport, Culture and the Media: The Unruly Trinity, Berkshire (Open University Press) 1999. 